\title{
Experimental Study on the Use of Thermoelectric Generators in Harvesting Human Body Heat
}

\author{
N. Rosli ${ }^{1 *}$, H. Mohamed ${ }^{2}$ \\ ${ }^{12}$ Department of Mechanical Engineering, Universiti Tenaga Nasional, Malaysia \\ *Corresponding author E-mail: suhailarosli4@gmail.com
}

\begin{abstract}
The objective of this work is to investigate the potential use of a thermoelectric generator (TEG) in harvesting human body heat at four body locations namely forehead, wrist, palm and calf for different human activities in Malaysia. Important parameters for this experiment are human body temperature, room temperature, output voltage, output resistance and output power. A healthy male and female perform a series of activities including resting, walking and running for 10 minutes in an indoor setting. Results indicate the relevant effects of temperature and movement on producing power from human body heat. Different parts of the body have different temperatures, thus creating different output powers. Results show that as the movement increases, the human body temperature and the amount of energy harvested also increase. It is also observed that forehead provides the highest amount of power generated during running activity. When the body temperature is above $35^{\circ} \mathrm{C}$, the TEG can generate power more than $5.0 \mathrm{~mW}$. The highest power generated during the experiment is $9.5 \mathrm{~mW}$. Even though Malaysia is known as a hot and humid region, TEGs can be feasibly utilized as a wearable device that converts human heat energy into electrical energy.
\end{abstract}

Keywords: Body heat; renewable energy; skin temperature; thermoelectric generators; wearable device.

\section{Introduction}

A thermoelectric generator, TEG, is a solid-state device that can convert thermal energy into electrical energy, which works similar to solar panels. Generally, thermoelectric uses the Seebeck effect as the conversion process where electricity can be produced from the temperature gradient across two conductors attached [1]. Electrons on the hot side of a TEG are more energized than on the cold side, resulting in migration of electrons from the hot side to the cold side when a temperature difference is applied.

The efficiency and power obtained from the TEG are not relatively high. Nonetheless, it is one of the alternative energy sources that can be implemented in various applications. Hence, many researchers have carried out investigations of energy harvesting from human body heat in recent years [2]. The thermoelectric generator system can harvest energy from human activities. It is estimated that a human body can create approximately $10-30 \mu \mathrm{W} / \mathrm{cm}^{2}$ of electrical power in an average climate [3]. Thermoelectric sensors, which are placed on the body, can produce an electrical voltage by finding the difference between the body temperature and the room temperature. Generally, a human body can release more than $100 \mathrm{~W}$ of energy wasted as heat. However, it is still limited by the Carnot efficiency to harvest the waste heat.

A previous study has shown that thermoelectric generators can be integrated into clothes, connecting the TEG technology with fashion sports equipment such as leg guards, sports tights, wristbands, and also headbands [4]. Another related work by Myers et al. has determined the effects of environmental and human factors on thermal energy generator performance for numerous conditions. The studied wearable TEG device was placed at the forehead and upper arm of human subjects while undergoing different activities in various environmental conditions. Results from the experiment show that the amount of power produced is different between individuals, activities performed and heat convection process with the environment [5].

A study by Francioso et al. [6] also reports that a temperature difference of about $9^{\circ} \mathrm{C}$ was obtained across a TEG when it was placed on human body skin while working at a room temperature of $24.8{ }^{\circ} \mathrm{C}$. The power generated was around $4.9 \mathrm{~mW}$. It is also observed that the thermoelectric performance can be improved by adding additional thick metal layers on both sides of the TEG. All these previous studies, however, are very limited to ambient temperatures within low and dry regions, ranging between 19 to $23{ }^{\circ} \mathrm{C}$. Malaysia, on the other hand, is situated in a tropical area where the daily air temperature fluctuates from a low of $24{ }^{\circ} \mathrm{C}$ up to $38{ }^{\circ} \mathrm{C}$. The country has quite high humidity, ranging from $70 \%$ to $90 \%$ throughout the year [7]. Since Malaysia is one of the hot and humid regions, it is highly essential for this work to investigate the practical usage of TEG in Malaysia because of the high ambient temperature, hence causing a small temperature difference between human body and room temperatures. This is a research gap that will be answered in this work.

This project focuses on four different body locations for the TEG to obtain relatively high output power. Important parameters that have been taken into accounts are room temperature, human body temperature, human activity, gender, connection modes and output power. 


\section{Experimental Setup}

\subsection{Design of Experiment}

This project focuses on extracting heat energy from four parts of the human body, which are forehead, wrist, palm, and calf. The energy-harvesting rate for different activities is one of the significant factors when harvesting heat. Therefore, this project also investigates the effects of three human activities, including resting, walking and running when performing the test for each body part. During the experiment, each activity was carried out for at least 10 minutes in an indoor setting. A healthy male participant weighs $70 \mathrm{~kg}$ with $170 \mathrm{~cm}$ tall (body mass index of $24.22 \mathrm{~kg} / \mathrm{m}^{2}$ ), and a female participant weighs $60 \mathrm{~kg}$ with $164 \mathrm{~cm}$ tall (body mass index of $22.31 \mathrm{~kg} / \mathrm{m}^{2}$ ) have volunteered as test participants. The measurement for each different body part was repeated for three times to obtain an average reading. Two type-K thermocouples were connected in series and securely attached to the top and bottom of the TEG. These attachments were made to accurately record the skin temperature and room temperature by using a dual channel type- $\mathrm{K}$ thermocouple thermometer. The temperature difference between the skin temperature (hot side) and room temperature (cold side) was then calculated. Figure 1 shows the illustration of the TEG placement at one of human body parts. Whereas, Figure 2 shows the experimental assembly of the TEG and other devices.

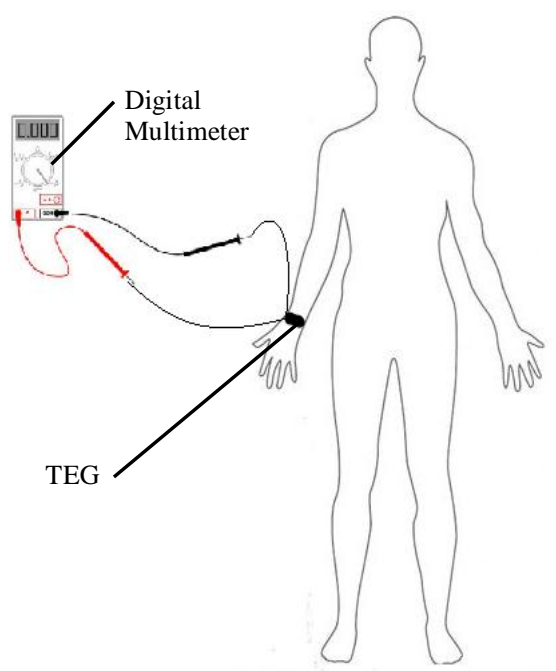

Fig.1: Illustration of energy harvesting at the human wrist.

Dual channel type-K thermometer thermocouple

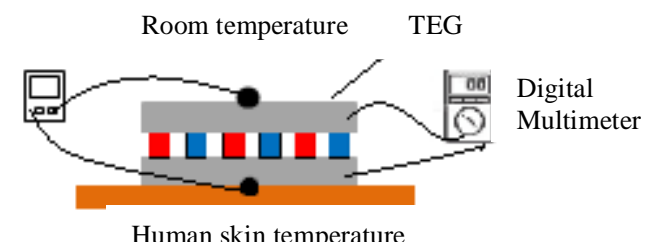

Fig 2: Experimental assembly of the TEG attached to the human skin.

\subsection{Experimental Procedure}

A small hole was made at the wristband and headband to place the TEG so that it is exposed to the room temperature. Next, the participants securely put on the wristband or headband and carefully adjusted the compression of the band as they found comfortable at their skin. The experiment was carried out between 10 am to $2 \mathrm{pm}$ to get a consistent temperature during the day. Temperatures for both sides (skin and room) were recorded before and after each activity to observe the temperature difference when the activity was carried out. The recorded data was also used to determine the temperature difference between both hot (skin) and cold sides for each part of the body.

Each activity was carried out for 10 minutes while the data for the TEG's voltage and resistance was recorded using a digital multimeter. When resting, participants simply sat on a chair. Whereas for walking and running, they performed the activities on a treadmill with speeds of $3.3 \mathrm{~km} / \mathrm{hr}$ and $7.3 \mathrm{~km} / \mathrm{hr}$ for walking and running, respectively. There was a rest time of 10 minutes after the participants completing the activity to reduce and stabilize the skin temperature and the heart rate. The device assembly at participants' body parts (wrist) is shown in Figure 3. For each temperature difference and activity, there will be a different amount of output power generated by the TEG system. Hence, once the voltage, $V$, and the resistance, $R$, are measured, the output power, $P$ can be calculated using Eq. (1): $r^{\prime \prime}=\frac{r^{x}}{m}$

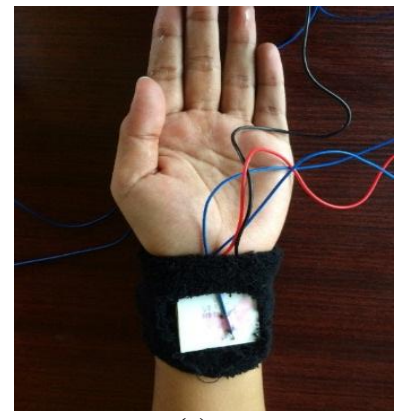

(a)

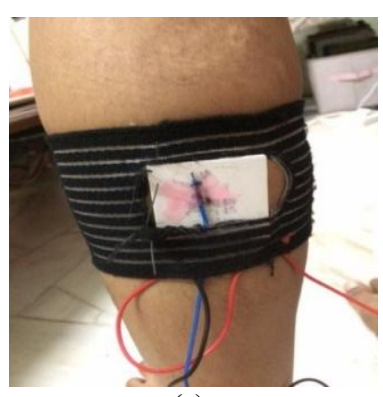

(c)

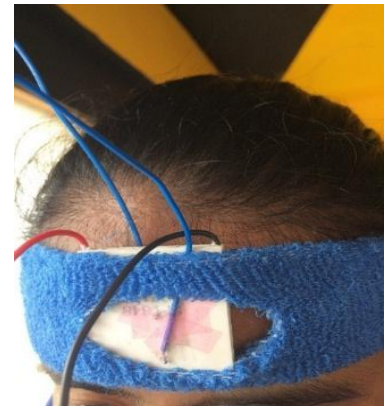

(b)

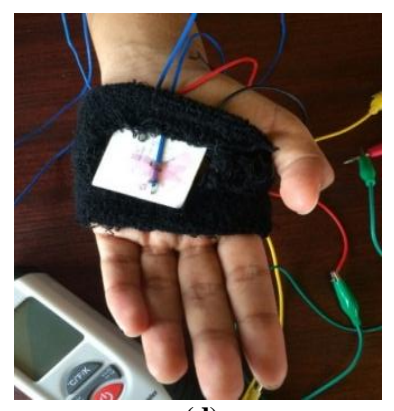

(d)
Fig 3: Device assembly a) at wrist; (b) forehead; (c) calf and (d) palm.

\section{Results and Discussion}

\subsection{Results for the Male Participant}

Figure 4 illustrates the comparison between the average temperature of hot side (human skin temperature) and the average temperature of cold side (room temperature) at four body locations namely forehead, wrist, palm and calf and three different activities.

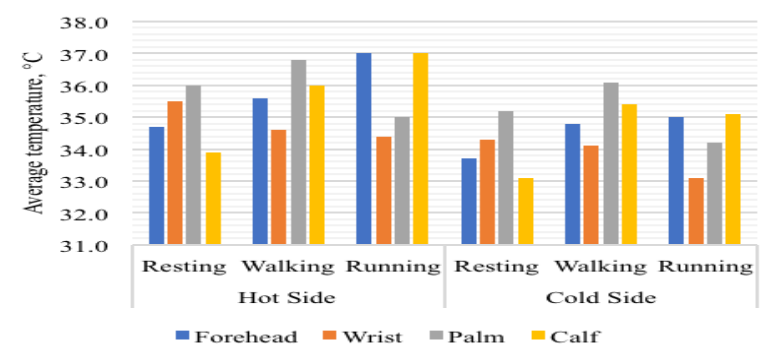

Fig 4: Comparison between the average temperature of the hot side and the average temperature of the cold side at different body locations for various activities for the male participant. 
Based on Figure 4, the graph shows the maximum and minimum average temperatures of the hot side that can be reached between $37{ }^{\circ} \mathrm{C}$ to $34.7^{\circ} \mathrm{C}$, depending on the body parts and the activities that were performed. It also shows that the trend for the average temperature of the cold side is similar to that of the hot side. However, the highest temperature of the cold side can only reach up to $36{ }^{\circ} \mathrm{C}$ for walking activity while the lowest temperature is below $34{ }^{\circ} \mathrm{C}$ for running activity. Figure 5 shows the average hot side temperature of the TEG before and after the activities for each of the body parts. It can be seen from the figure that the temperature for every body part increases after completing the activities.

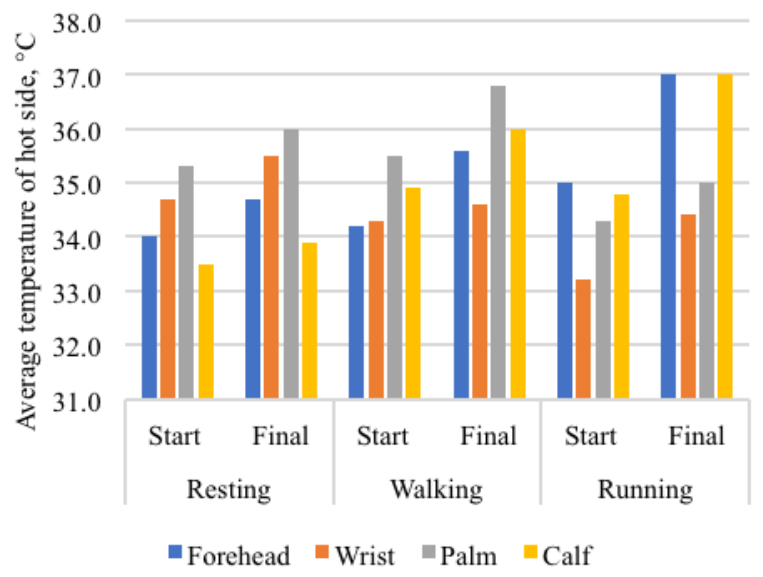

Fig 5: Comparison of the average temperature of the hot side before and after completing the activities at different body locations for the male participant.

As shown in Figure 5, palm surprisingly gives the highest temperature than calf, wrist and forehead. The palm temperature could reach over $36{ }^{\circ} \mathrm{C}$ when resting or walking. However, the forehead and calf provide the highest temperature in the running, which is about $37^{\circ} \mathrm{C}$. Since the experiment was conducted in a tropical region, each body location shows a small temperature difference after completing all the activities.

Figure 6 shows that palm has generated the highest power, which is about $9.5 \mathrm{~mW}$ for walking activity. This is due to the low average output voltage and low average output resistance acquired during the experiment, resulting in higher power being generated compared to other body parts. The calf also produces a high amount of power when performing walking activity compared to resting and running. For the running activity, forehead shows the highest amount of power generated. The output power increases as the activity changes from resting to running. Unlike forehead, the power trend for wrist is opposite, in which the power generated is decreasing from resting to running.

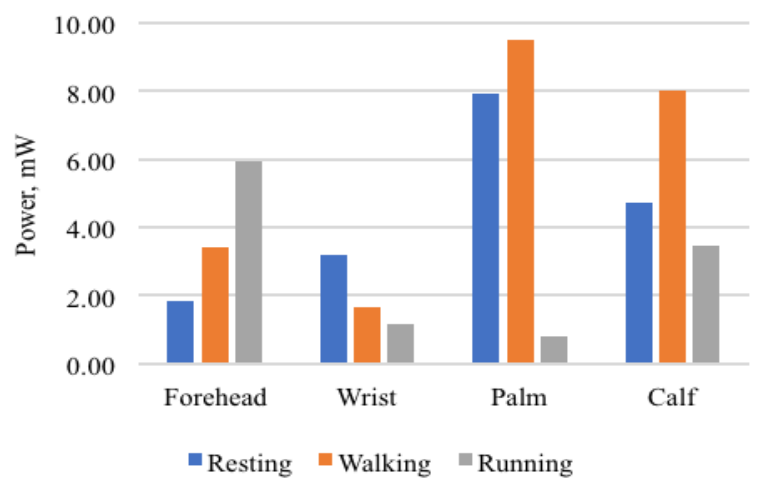

Fig 6: Comparison of power generated at different body locations for various activities for the male participant.

\subsection{Results for the Female Participant}

Figure 7 shows the maximum and minimum average temperatures of the hot side that can be reached up to $35{ }^{\circ} \mathrm{C}$ and as low as 30 ${ }^{\circ} \mathrm{C}$, depending on the body parts and activities performed. It also shows that there is a small temperature difference between the average temperature of the cold side and the average temperature of the hot side. When resting, the average hot side temperatures for four body parts show a very similar reading throughout the experiment, which is approximately $30{ }^{\circ} \mathrm{C}$. Interestingly, the average temperature of the cold side is also around $30{ }^{\circ} \mathrm{C}$ when resting, regardless of body parts.

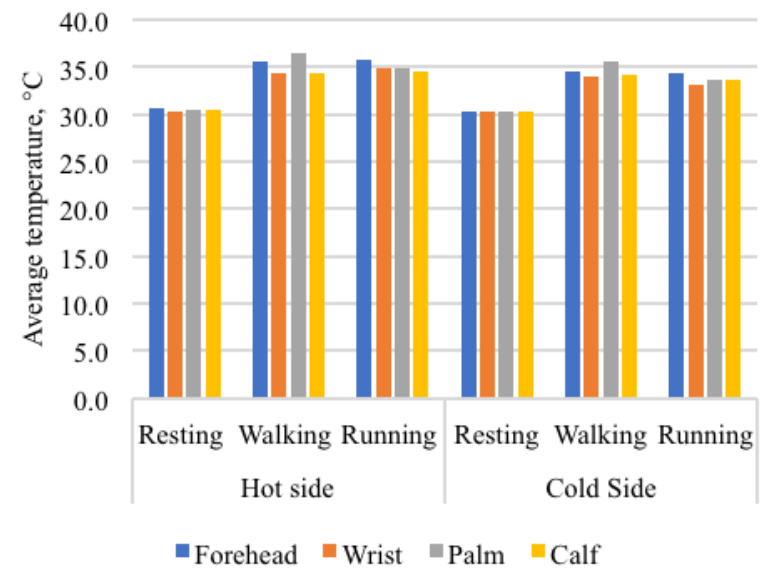

Fig 7: Comparison between the average temperature of the hot side and the average temperature of the cold side at different body locations for various activities for the female participant.

As shown in Figure 8, the average temperature for each body part slightly rises after completing the activities. Resting activity has the smallest temperature rise, which is about $0.1{ }^{\circ} \mathrm{C}$. The palm temperature presents the highest hot temperature for walking, while the highest temperature from running is at the forehead. Both temperatures are over $35{ }^{\circ} \mathrm{C}$. Figure 9 compares the power generated at different body locations for the three activities.

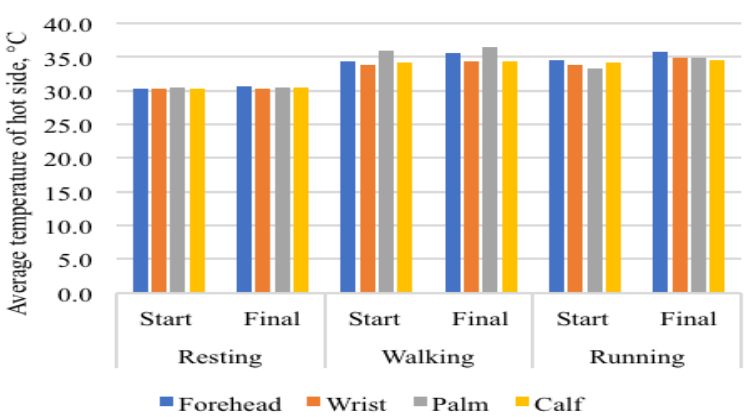

Fig 8: Comparison of the average temperature of the hot side before and after completing the activities at different body locations for the female participant.

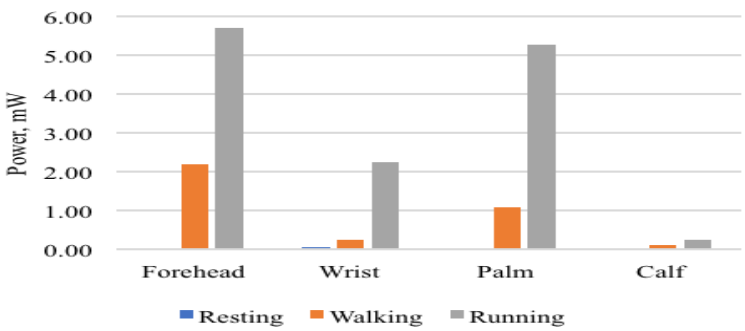


Fig 9: Comparison of power generated at different body locations for various activities for the female participant.

Based on Figure 9, it can be seen that the power generated at the forehead is still dominant for walking and running activities where $2.1 \mathrm{~mW}$ for walking and $5.7 \mathrm{~mW}$ for running. In comparison to the wrist and palm, calf provides the lowest power generated which is below $0.5 \mathrm{~mW}$ for each of the activities. Although the trends for the average output voltage and power are identical where those values rise as the activity changes from resting to running, the energy harvested from resting is significantly small. The power generated is about $0.01 \mathrm{~mW}$. It can be concluded from female participant results that an increase in the rate of energy harvesting can be potentially achieved by increasing the movement. This finding is in agreement with Ref. [5].

\subsection{Results Comparison between Male and Female Participants}

The average hot side temperatures of male and female participants are relatively similar for all activities (over $34^{\circ} \mathrm{C}$ ), except for the female's resting temperature. The resting temperature stays around $30{ }^{\circ} \mathrm{C}$ regardless of body parts as shown in Figure 10. When comparing male and female results, it is shown that most male's average temperatures are higher than those of female. This is reasonably true since various studies have found that male's hand temperatures tend to be higher than female's hand temperature due to the underlying physiological variations such as weight, height and proportions of a body that may influence the capability of male and female to maintain heat [8].

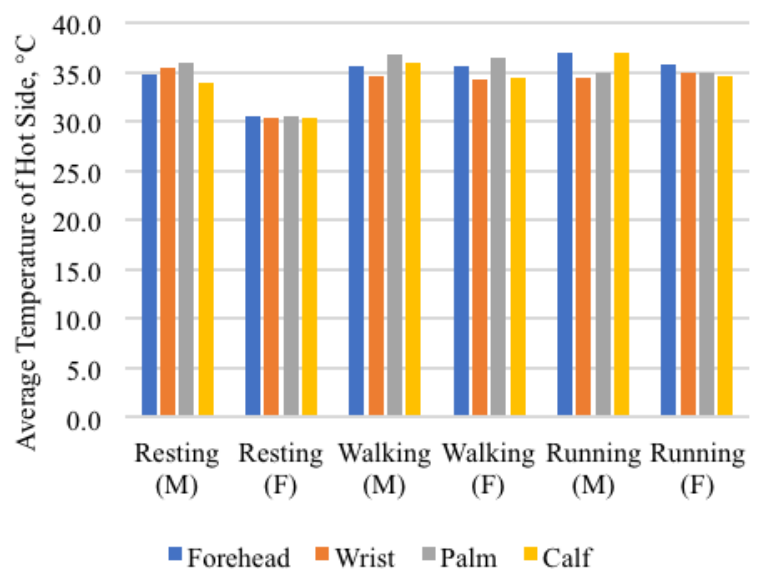

Fig 10: Comparison of the average hot side (skin) temperature between male and female participant at different body locations for various activities.

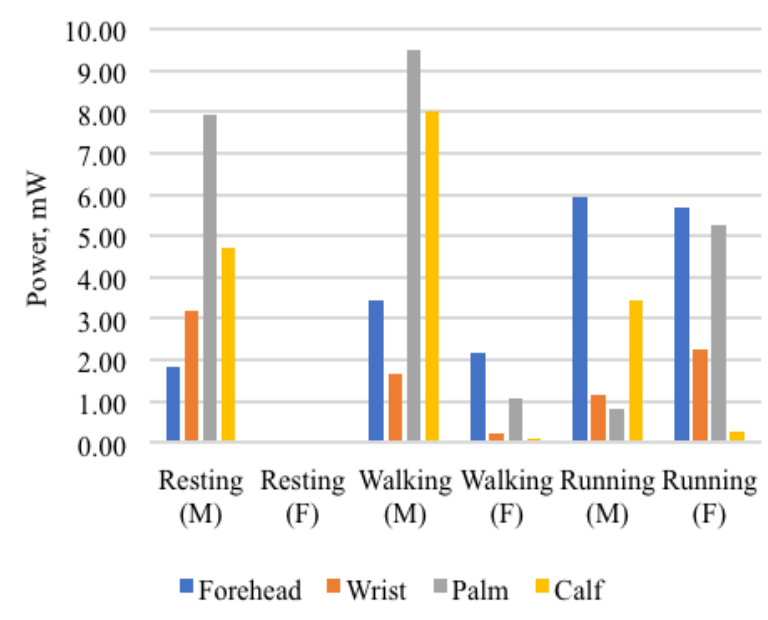

Fig 11: Comparison of power generated between male and female participant at different body locations for various activities.

Figure 11 shows the differences in power generated from male and female participants at each body part for resting, walking and running. Powers created when resting for the female participant are too small regardless of body parts, which are about $0.01 \mathrm{~mW}$. It can also be seen that the power generated at the wrist or calf for female's walking activity has the lowest value, which is below than $0.05 \mathrm{~mW}$. In contrast, the power generated for the male participant shows the highest reading in every activity, which is generally over $1.5 \mathrm{~mW}$. Nonetheless, for running, the lowest power generated is actually at palm, approximately $0.8 \mathrm{~mW}$. Body parts that can generate the maximum power for each activity are identified and tabulated in Table 1 and Table 2 for male and female participants, respectively.

Table 1: Maximum power generated with the related body part for each activity performed by the male participant

\begin{tabular}{|c|c|c|}
\hline Body Parts & Activity & Power, mW \\
\hline Palm & Resting & 7.93 \\
\hline Palm & Walking & 9.50 \\
\hline Forehead & Running & 4.49 \\
\hline
\end{tabular}

Table 2: Maximum power generated with the related body part for each activity performed by the female participant

\begin{tabular}{|c|c|c|}
\hline Body Parts & Activity & Power, $\mathrm{mW}$ \\
\hline Wrist & Resting & 0.04 \\
\hline Forehead & Walking & 2.18 \\
\hline Forehead & Running & 5.69 \\
\hline
\end{tabular}

Referring to Table 1, it shows that for resting and walking, palm has the highest power generated as compared to other body parts while for running, the power harvested from the forehead is the largest. On the other hand, it can be observed in Table 2, for the female participant, the location of the maximum power for walking and running is at the forehead. While for resting, wrist offers the most significant output power, though it is still the smallest one compared to the power that can be harvested from the other two activities. For the running activity, both male and female participants have forehead as their optimal location for harvesting energy, which is mainly due to the highest hot side temperature at forehead (Figure 10).

Based on the results, male and female participants generate different power depending on body parts and activities. For the male participant, the palm is likely to be the best part to generate power while for the female participant, the ideal part to generate power is forehead. The vital pattern discovered is that the amount of power can be harvested increases as the body movement increases. This holds true only for our female results, confirming the finding by Myer et al. [5]. A study by Wang et al. further explains that skin temperature and local blood flow are interconnected to each other, increasing skin temperature when the 
movement increases [9]. Deng et al. also has explained that the reason different body parts have different skin temperature is that of the variation in heat flow and the thermal resistance of different parts of a body [4].

\subsection{Factors that May Affect the Results and Observations}

Humans are a warm-blooded species, and they are very efficient in balancing temperature to keep their body temperature within a safe range despite external thermal changes [10]. The skin temperature is regularly unbalanced and fluctuating, ranging from $31{ }^{\circ} \mathrm{C}$ to $35^{\circ} \mathrm{C}$. The cause of body temperature to be balanced is due to the hypothalamus where it is located in the area of the brain that performs as the body's thermostat. The hypothalamus stabilizes heat gain against heat loss through some physical and behavioral mechanisms such as adjusting skin blood flow [11]. The blood flow in a body depends on different heat transfer processes and the effective insulation inside the skin [12].

Most of the heat generated in the body is originated in the deep organs such as the heart, brain, liver and the skeletal muscles during exercise. There are two heat transfer processes within the body that need to be considered, namely the heat transfer from the upper body parts (e.g., forehead) to the rest of the body and from the inner body core to the skin [13]. Heat is transferred inside the body by conduction through the tissues and by convection through the blood flow, in which the blood is transporting the heat from warmer tissues to colder tissues [13][14]. The heat is carried through the blood vessels where it is circulated beneath the skin throughout the human body until it flows up to the head and torso, causing the upper body to usually have higher skin temperature compared to the lower body [15].

Even though skin temperature varies widely across the body surface, it is also largely influenced by environmental conditions [16]. In the morning, the body temperature is low due to the body just waking up from sleeping. The temperature will gradually increase after food intake and routine activities until the night. Since this experiment was conducted at 10 am and at a room temperature, it can be seen from the results that the participants' skin temperatures before starting the activities did not significantly increase. Therefore, the temperature difference between the participants' skin temperature and the room temperature was small.

It was proven that a male has a higher temperature compared to a female due to the physiological differences between both genders. Also, a male generally has more muscle mass than a female has, which will help them to produce more heat [8]. Apart from that, body metabolism of male and female is also one of the factors that both genders have different temperature behavior. Studies show that those with higher metabolic rate might have a higher body temperature, in contrast to those with slower metabolic rate who might have a lower body temperature [12]. Other factors that may influence the metabolic rate are body size, body composition, genetic, hormonal levels, lifestyle, and health [17].

Based on the results obtained, for both genders, output power increases as the movement increases, especially when the TEG is placed at the forehead. The reason is that the temperature is most stable at the forehead and it always maintains relatively high [16]. Another relevant factor that affects the output power from harvesting human body heat is the squeezing experience at all four body parts. This squeeze limits the arteries, hence slowing down the blood flow supply to the brain, upper and lower limbs [12].

Brink [12] has reported that at first, the TEG was at room temperature and after it was in contact with the skin, which is the hot side, a rapid rise of the temperature due to significant temperature difference resulting the output voltage to generate at the highest peak in a short time. After a few times, the output voltage declined as the TEG warmed up with skin temperature due to low heat transfer rate as shown in Figure 12.

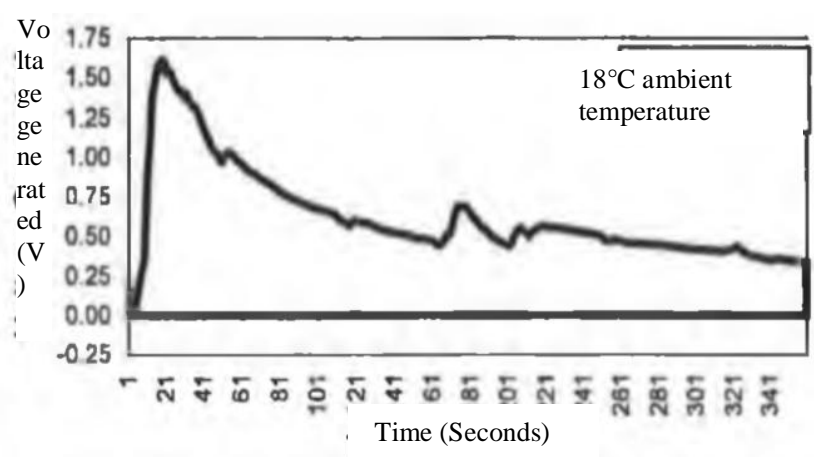

Fig 12: The experimental result of the voltage generated at neck using thermoelectric collar. Reproduced from Ref. [12].

Brink also observed that the available heat energy reduces as the blood flow continuously carry away heat from the skin too, causing a decline in the temperature difference after a certain period [12]. In addition, the cause of the drop in the TEG effectiveness is also due to the absence of heat sinks to remove the heat away from the TEG, thus trapping the waste heat inside the TEG. It is also important to note that since the irregular contour of a body surface, the TEG was not in full contact with the skin, especially at palm and wrist. Therefore, the effectiveness of the TEG again could not be achieved.

Additionally, the calculated output power depends on the output voltage and the internal resistance. This relationship varies depending on the changing temperature difference from the experiment. Since the TEG is connected in parallel, the result obtained is dependent on the individual TEG voltages and internal resistances. The internal resistance of the TEG is increasing with the rising temperature. The internal resistance is defined as the sum of the TEG resistance and the resistance due to the temperature change [18].

\section{Conclusion}

In conclusion, this study has determined the impacts of environmental and human factors on the TEG performance for various movements, namely resting, walking and running. As the movement increases, the temperature of the hot side, which is the skin temperature, also increases. The experimental results show that when the skin temperature reaches above $35^{\circ} \mathrm{C}$, the TEG can harvest power more than $5.0 \mathrm{~mW}$. Forehead offers the highest amount of power generated when running, based on the male and female experimental results. However, compared to other parts and activities, the highest output power was generated at the male's palm when resting, which is $9.5 \mathrm{~mW}$. Most values obtained are comparable to the value reported by Francioso et al. [6], which is around $4.9 \mathrm{~mW}$.

Above all, the performance of the TEG is promising. Although the output power generated is small, it still can light up an LED light, which consumes about $4 \mathrm{~mW}$ power. Other small applications such as a muscle simulator $(1.3 \mathrm{~mW})$, neurostimulator $(50 \mu \mathrm{W})$, hearing aid $(1 \mathrm{~mW})$ and pacemaker $(8 \mu \mathrm{W})$ can also benefit from the use of the wearable TEG. Hence, it can be concluded that it is feasible to utilize TEGs for harvesting human body heat in Malaysia. Nevertheless, many factors need to be taken into consideration to boost the energy harvesting performance. The output power can be improved by installing a voltage booster in the electrical connection or by adding the quantity of the TEGs, instead of using a single TEG only. It is also highly recommended to increase the number of participants for future improvement to get consistent results. 


\section{Acknowledgment}

The author would like to express sincere appreciation for the College of Engineering, Universiti Tenaga Nasional for sponsoring the research project under the UNITEN Start-up grant Bold 2025 project code RJO10289176/B/1/2017/20.

\section{References}

[1] M. Wahbah, M. Alhawari, B. Mohammad, H. Saleh and M. Ismail, "Characterization of Human Body-Based Thermal and Vibration Energy Harvesting for Wearable Devices," Emerging and Selected Topics in Circuits and Systems, vol. 4, no. 3, 2014.

[2] M. Jaworski, M. Bednarczyk and M. Czachor, "Experimental Investigation of Thermoelectric Generator (TEG) with PCM module," Applied Thermal Energy, vol. 96, pp. 527-533, 2015.

[3] H.-P. Wong and Z. Dahari, "Human Body Parts Heat Energy Harvesting Using Thermoelectric Module," IEEE Journal, 2015.

[4] F. Deng, H. Qiu, J. Chen, L. Wang and B. Wang, "Wearable Thermoelectric Power Generators Combined with Flexible Supercapacitor for Low Power Human Diagnosis Devices," Industrial Electronics, vol. 64, pp. 1477-1485, 2017.

[5] Myers, R. Hodges, Jur, and S. Jesse, "Human and Environmental Analysis of Wearable Thermal Energy Harvesting," Energy Conversion and Management, vol. 143, pp. 218-226, 2017.

[6] L. Francioso, C. D. Pascali, V. Sglavo, A. Grazioli, M. Masieri and P. Siciliano, "Modelling, Fabrication and Experimental Testing of a Heat Sink Free Wearable Thermoelectric Generator," Energy Conversion and Management, vol. 145, pp. 204-213, 2017.

[7] N. Makaremi, E. Salleh, M. Z. Jaafar and A. Ghaffarian Hoseini, "Thermal Comfort Conditions of Shaded Outdoor Spaces in Hot and Humid Climate of Malaysia," Building and Environment, vol. 48 , pp. 7-14, 2012

[8] W. Nicole, "Healthy Life," 22 July 2016. Available online: https://www.simplysupplements.co.uk/healthylife/generalhealth/body-temperature-how-it-differs-for-men-and-women. [Accessed 201711 14].

[9] D. Wang and N. Zhu, Physiology, Beijing, China: People's Medical Publishing, 2013.

[10] W. Nicole, "Simply Supplements," Healthy Life, 22 July 2016. Available online: https://www.simplysupplements.co.uk/healthylife/generalhealth/body-temperature-how-it-differs-for-men-and-women. [Accessed 27 December 2017].

[11] Shoemaker and A. L, "What's Normal? Temperature, Gender, and Heart Rate," Journal of Statistics Education, vol. 4, p. 2, 2004.

[12] R. Brink, Development of Miniature Personal Thermoelectric Generator, Dublin: Dublin City University, 2004.

[13] V. Allen, "78 Steps Health," 28 September 2016. [Online]. Available: https://www.78stepshealth.us/skeletal-muscle-2/bodytemperatures-and-heat-transfer-in-the-body.html. [Accessed 27 December 2017].

[14] H. Alo, "Temperature Regulation by Skin," LinkedIn Corporation, 2014.

[15] H. Zhu, H. Wang, Z. Liu, G. Kou, C. Li and D. Li, "Experimental Study on The Variations in Human Skin Temperature Under Simulated Weightlessness," Building and Environment, vol. 117, pp. 135-145, 2017.

[16] M. Garcia-Souto and P. Dabnichki, "Skin Temperature Distribution and Thermoregulatory Response During Prolonged Seating," Building and Environment, vol. 69, pp. 14-21, 2013.

[17] M. T. Murray, "Share Care," 1 December 2007. [Online]. Available: https://www.sharecare.com/health/how-the-metabolicprocess-works/factors-influence-basal-metabolic-rate. [Accessed 29 December 2017].

[18] Montecucco, J. Siviter and A. R. Knox, "The Effect of Temperature Mismatch on Thermoelectric Generators Electrically Connected in Series and Parallel," Applied Energy, vol. 123, pp. 47-54, 2014. 